\title{
Comparing the effect of cardiac biomarkers on the outcome of normotensive patients with acute pulmonary embolism
}

\author{
Yaser Jenab, Marzieh Pourjafari, Maryam Sotoudeh, Masoumeh Lotfi-tokaldany, \\ Nasrin Etesamifard, Shapour Shirani, Arash Jalali, Younes Nozari, Hamidreza Poorhosseini \\ Tehran Heart Center, Tehran University of Medical Sciences, Tehran, Iran
}

\begin{abstract}
Acute pulmonary embolism (PE) is a cardiovascular challenge with potentially fatal consequences.

This study was designed to observe the association of novel cardiac biomarkers with outcome in this setting. In this prospective study, from 86 patients with a confirmed diagnosis of $\mathrm{PE}, 59$ patients met the inclusion criteria ( 22 men, 37 women; mean age, $63.36 \pm 15.04$ y). The plasma concentrations of N-terminal pro-brain natriuretic peptide (NT-proBNP), growth differentiation factor-15 (GDF-15), heart-type fatty acid-binding protein (H-FABP), tenascin-C, and D-dimer were measured at the time of confirmed diagnosis. The endpoints of the study were defined as the short-term adverse outcome and long-term all-cause mortality.

Totally, 11.8\% (7/59) of the patients had the short-term adverse outcome. The mean value of $\operatorname{logNT}$-proBNP was $6.40 \pm 1.66 \mathrm{pg} / \mathrm{ml}$. Among all the examined biomarkers, only the mean value of logNT-proBNP was significantly higher in the patients with the short-term adverse outcome $(7.88 \pm 0.67$ vs $6.22 \pm 1.66 \mathrm{pg} / \mathrm{ml}$; OR, $2.359 ; 95 \% \mathrm{CI}, 1.037$ to 5.367 ; $\mathrm{p}=0.041$ ). After adjustment, a threefold increase in the short-term adverse outcome was identified (OR, 3.239; $95 \% \mathrm{CI}, 0.877$ to 11.967 ; $\mathrm{p}=0.078)$. Overall, $18.64 \%$ (11/59) of the patients had expired by the long-term follow-up. Moreover, adjustment revealed an evidence regarding association between increased logNT-proBNP levels and longterm mortality (HR, 2.163; 95\%CI, 0.910 to 5.142 ; $\mathrm{p}=0.081$ ).

Our study could find evidences on association between increased level of NT-proBNP and short-term adverse outcome and/or long-term mortality in PE. This biomarker may be capable of improving prediction of outcome and clinical care in non-high-risk PE.
\end{abstract}

Corresponding author: Associate Prof. Yaser Jenab, Tehran Heart Center, North Kargar Street, Tehran 1411713138, Iran.

Tel. $+98.21 .88029600-60$ - Fax +98.21 .88029257 .

E-mail: jenab@razi.tums.ac.ir

Key words: Acute pulmonary embolism; biomarker; N-terminal pro-brain natriuretic peptide.

Received for publication: 24 August 2016

Accepted for publication: 31 January 2017

(c) Copyright Y. Jenab et al., 2017

Tipografia PI-ME Editrice, Italy

Monaldi Archives for Chest Disease 2017; 87:767

doi: 10.4081/monaldi.2017.767

This article is distributed under the terms of the Creative Commons Attribution Noncommercial License (by-nc 4.0) which permits any noncommercial use, distribution, and reproduction in any medium, provided the original author(s) and source are credited.

\section{Introduction}

Acute pulmonary embolism (PE) is a life-threatening clinical challenge. Acute complications of PE affect approximately 10\% of patients and the mortality rate varies from greater than $25 \%$ in hemodynamically unstable patients, to $3-15 \%$ for stable patients with right ventricular (RV) dysfunction [1].

In the prognostic assessment of PE, the relative standing of clinical symptoms versus clinical prognostic rules, biomarkers, echocardiography, and computed tomography (CT) scan is still a matter of debate $[2,3]$. In recent years, different markers of RV dysfunction, myocardial injury, and noncardiac laboratory biomarkers have been studied and suggested to have prognostic roles in acute PE [4]. However, there are limited data on the use of different biomarkers in predicting mortality in patients with acute PE. In patients with acute PE, elevated concentrations of brain natriuretic peptide (BNP) or N-terminal pro-brain natriuretic peptide (NT-proBNP), circulating heart-type fatty acid-binding protein (H-FABP), tenascin-C, growth differentiation factor-15 (GDF-15), and D-dimer on admission have been shown to be helpful to identify patients at risk of death or adverse clinical outcome during the acute phase of PE [4-6].

In the present study, we investigated whether the levels of NT-proBNP, GDF-15, H-FABP, tenascin-C, or D-dimer have associations with shortterm adverse events and/or long-term mortality in normotensive patients with acute PE.

\section{Materials and Methods}

\section{Study population}

This prospective study was carried out from April 2013 to April 2014 in the emergency department of a tertiary care university hospital. Consecutive patients with confirmed acute PE diagnosis by pulmonary computed tomography angiography (CTA) were included in the study.

The aim of the present study was to compare the association between a panel of biomarkers and outcome in acute PE. Since the levels of biomarkers can be influenced by concomitant underlying diseases such as heart failure, renal failure, and malignancy which can render the interpretation of the markers results challenging [7]. Accordingly, we excluded patients with the following criteria: hemodynamic instability at presentation, (minimum systolic blood pressure of $90 \mathrm{mmHg}$ ); history of heart failure; history of malignancy; glomerular filtration rate below 30 on admission; reduced left ventricular ejection fraction (ejection fraction <40\%); delayed pulmonary CTA (72 h after admission); history of PE; or complication by acute PE during hospitalization.

\section{Study protocol}

All the patients were managed according to the routine clinical assessment at the Emergency Department of our center. In the acute 
phase the patients were treated with conventional therapeutic doses of unfractionated or low-molecular-weight heparin. The application of thrombolytic therapy was at the physician's discretion. All the patients also received warfarin throughout the clinical course.

The study protocol was approved by local Ethics Committee of our hospital. Written informed consent was obtained from the patients or their family members and data on the variables were recorded on a standardized form.

\section{Definitions of the outcomes}

The primary end point of the study was the short-term adverse events defined as death or any of the following complications: cardiopulmonary resuscitation, hemodynamic instability (systolic blood pressure $<90 \mathrm{~mm}$ $\mathrm{Hg}$ or drop of systolic blood pressure by $>40 \mathrm{~mm} \mathrm{Hg}$ for $>15 \mathrm{~min}$ with signs of end-organ hypoperfusion), inotrope use, thrombolysis and/or thrombectomy, and the use of mechanical ventilation within 1 month of admission. The secondary end points of the study were: a) all-cause longterm mortality defined as death due to any cause after the diagnosis of PE up to the end of the study; b) right ventricular dysfunction association with different biomarker levels. RV dysfunction was defined as the dilatation of the RV (end-diastolic diameter $>35 \mathrm{~mm}$ or a right/left ventricular diameter ratio $\geq 1$ in the apical four-chamber view) or pulmonary artery pressure more than $40 \mathrm{mmHg}$ on transthoracic echocardiography performed within $48 \mathrm{~h}$ of diagnosis.

\section{Patient follow-up}

The patients were followed up in the outpatient clinic. For the shortterm follow-up, at discharge, the patients were instructed to return for a clinical check 30 days after their admission. If a patient missed a followup visit, the data coordinator would make a telephone call. The longterm follow-up was performed through hospital visits if the patient was available. At the end of the follow-up, all the patients missing the hospital visits were contacted through phone calls by the data coordinator, who was blinded to the outcome and results of the biomarkers. All the patients underwent a long-term follow-up for a median of 17.3 months (range between 1.2 and 24.4 months), by the end of the study.

\section{Measurement of the samples}

All the measurements and biochemical tests were performed using standard laboratory techniques. All the investigators who determined the biomarker levels were blinded to the baseline parameters or the clinical course of the patients and the biomarker results were not used for patient management.

\section{Biochemical analysis}

Peripheral venous blood specimens from antecubital vein were centrifuged within $1 \mathrm{~h}(3000 \mathrm{~g}$ for $5 \mathrm{~min})$, and the sera were stored at $-80^{\circ} \mathrm{C}$ until analysis.

NT-proBNP was determined via the fully-automated electrochemiluminescent assay using the Elecsys 2010 (Roche Diagnostics, Indianapolis, IN, USA) with the sandwich principle and a total duration of 18 min. The patients' sample was mixed with a biotinylated monoclonal NT-proBNP specific antibody and a monoclonal NT-proBNP-specific antibody labeled with a ruthenium complex antibody. Functional sensitivity was 50 picograms per milliliter $(\mathrm{pg} / \mathrm{ml})$, with a cutoff value of $125 \mathrm{pg} / \mathrm{ml}$. GDF-15was assessed using a BioVendor Human GDF-15/MIC-1 ELISA kit (Czech Republic) with a sandwich biotin-labeled antibody. The sensitivity was $22 \mathrm{pg} / \mathrm{ml}$, and the intra- and inter-assay precision (CV values) was 6.8 and 9\%, respectively. H-FABP was measured quantitatively using the point-of-care MyoCheck H-FABP (quant.) (Concile GMBH, Freiburg,
Germany) Automatic measurement was carried out using the chromatographic sandwich immunoassay, with a cutoff value of 7 nanograms per milliliter (ng/ml). Tenascin-C was quantified using the tenascin-C Large (FNIIIB) ELISA kit IBL (Hamburg, Germany) via the sandwich immunoassay. The measurement range was between 0.38 and $24 \mathrm{ng} / \mathrm{ml}$, according to the manufacturer's instructions. The sensitivity was 0.01 $\mathrm{ng} / \mathrm{ml}$, and the intra- and inter-assay precision (CV values) was 6.6 and $7 \%$, correspondingly. The D-dimer test principle was the particle-enhanced immunoturbidimetric assay using the Tina-quant D-Dimer Gen.2 kit and COBAS INTEGRA400 plus instrument (Roche Diagnostics). A measuring range between 0.15 and 9.00 micrograms of fibrinogen equivalent units per milliliter was used in the preparation of the original Asserachrom standard. The sensitivity was $100 \%$; negative predictive value, $100 \%$; specificity, 50.4\%; positive predictive value, $40.5 \%$; and failure rate, $0 \%$. Performance of the whole assay was monitored using two levels of normal and abnormal control with a commercial serum control kit.

\section{Statistical analysis}

The continuous variables were described as mean and standard deviation (SD) or median with $25^{\text {th }}$ and $75^{\text {th }}$ percentiles and were compared between the complicated and noncomplicated outcome groups as well as the RV dysfunction groups applying the independent-samples $t$-test or the Mann-Whitney U test. Because of the wide range of NT-proBNP and GDF15 , the logarithm of these variables was considered in the analyses. The categorical variables were presented as number and percentage and were compared between the aforementioned groups using the chi-square or the Fisher exact test. Variables with a P value less than 0.2 in the univariate analysis were considered as potential confounders. The effect of the biomarkers on the short-term and long-term outcomes were adjusted for the detected potential confounders using the logistic regression and the Cox proportional hazards model, respectively. The effect of the biomarkers on the short-term and long-term outcomes was reported through odds ratio (OR) and hazards ratio, with a 95\% confidence interval (CI), correspondingly. All the statistical analyses were performed using IBM SPSS statistics for Windows, ver. 22.0 (IBM Corp., Armonk, NY, USA).

\section{Results}

\section{Patients' background and short-term adverse events}

During the course of the study, 86 consecutive patients with a confirmed diagnosis of normotensive acute PE by pulmonary CTA were enrolled in the study. Twenty-seven patients were excluded from the study: twelve patients due to a history of previous $\mathrm{PE}$, five due to heart failure, five due to underlying or history of malignancy, one due to endstage chronic kidney disease, two due to PE complications during admission, one due to another accompanying diagnosis (acute myocardial infarction), and one due to delayed sampling. A total of 59 eligible in-patients managed at the physician's discretion were analyzed.

The baseline clinical characteristics of the study patients are presented in Table 1. The mean age of study population was $63.36 \pm 15.04 \mathrm{y}$. The mean value for respiratory rate and systolic blood pressure were $25.79( \pm 5.96)$ and $137.95( \pm 21.24)$. There was no statistically significant difference between complicated and uncomplicated patients within one month of acute PE in terms of age $(64.86 \pm 12.199$ vs $63.15 \pm 15.477$, $\mathrm{p}=0.777)$; respiratory rate $(28.67 \pm 6.408$ vs $25.43 \pm 5.871, \mathrm{p}=0.218)$; and systolic blood pressure $(144.00 \pm 25.502$ vs $137.13 \pm 20.756, \mathrm{p}=0.422)$. Moreover, the mean value for left ventricular ejection fraction for study patients was $55.02 \%( \pm 0.03)$. No significant difference was found between two complicated and uncomplicated study subgroups based on the left ventricular ejection fraction $(54.71 \pm 0.756$ vs $55.06 \pm 3.22, \mathrm{p}=0.777)$. 
Table 1. General characteristics of patients with acute normotensive pulmonary embolism.

\begin{tabular}{|c|c|c|c|c|}
\hline Variables & $\begin{array}{c}\text { Patients } \\
(n=59)\end{array}$ & $\begin{array}{l}\text { Complicated } \\
\text { short-term outcome } \\
\quad(n=7)\end{array}$ & $\begin{array}{l}\text { Non-complicated } \\
\text { short-term outcome } \\
(\mathrm{n}=52)\end{array}$ & p-value \\
\hline Gender (female) (n, \%) & $37(62.71)$ & $4(57.1)$ & $33(63.5)$ & $<0.999$ \\
\hline \multicolumn{5}{|l|}{ Signs and symptoms on admission } \\
\hline Heart rate $\geq 110 \mathrm{bpm}$ & $10(16.9)$ & $2(28.6)$ & $8(15.4)$ & 0.338 \\
\hline Transcutaneous $\mathrm{O}_{2}$ saturation $<90 \%$ & $12(20.3)$ & $3(42.9)$ & $9(17.3)$ & 0.141 \\
\hline Dyspnea & $50(86.2)$ & $7(100)$ & $43(84.3)$ & 0.577 \\
\hline Chest pain & $14(28.0)$ & $1(16.7)$ & $13(29.5)$ & 0.663 \\
\hline syncope & $4(7.5)$ & $0(0)$ & $4(8.5)$ & $<0.999$ \\
\hline \multicolumn{5}{|l|}{ Risk factors and comorbidities (n, \%) } \\
\hline Diabetes & $9(15.3)$ & $1(14.3)$ & $8(15.4)$ & $<0.999$ \\
\hline Hypertension & $33(55.9)$ & $5(71.4)$ & $28(53.8)$ & 0.449 \\
\hline Hyperlipidemia & $14(23.7)$ & $1(14.3)$ & $13(25.0)$ & $<0.999$ \\
\hline Current smoker & $12(20.3)$ & $3(42.9)$ & $9(17.3)$ & 0.141 \\
\hline Surgery within 4 weeks & $10(16.9)$ & $2(28.6)$ & $8(15.4)$ & 0.338 \\
\hline Travel history $>4$ hours by air/land & $12(23.1)$ & $1(16.7)$ & $11(23.9)$ & $<0.999$ \\
\hline History of DVT & $3(5.1)$ & $0(0)$ & $3(5.8)$ & $<0.999$ \\
\hline Immobilization (3 days or more) & $16(27.1)$ & $2(28.6)$ & $14(26.9)$ & $<0.999$ \\
\hline Chronic pulmonary disease & $2(3.4)$ & $0(0)$ & $2(3.8)$ & $<0.999$ \\
\hline Obesity & $11(18.6)$ & $1(14.3)$ & $10(19.2)$ & $<0.999$ \\
\hline \multicolumn{5}{|l|}{ Echocardiographic parameters } \\
\hline Right ventricular dysfunction & $31(52.5)$ & $6(85.7)$ & $25(48.1)$ & 0.106 \\
\hline \multicolumn{5}{|c|}{ Pulmonary Multislice computed tomography findings } \\
\hline Segmental PE & $13(22.0)$ & $0(0)$ & $13(25.0)$ & 0.330 \\
\hline Pulmonary infarction & $11(19.3)$ & $1(14.3)$ & $10(20.0)$ & $<0.999$ \\
\hline
\end{tabular}

Data are presented as number (percent), mean \pm standard deviation or medians $\left(25^{\text {th }}\right.$ to $75^{\text {th }}$ percentile).

\section{Outcomes}

The short-term adverse events were observed in $11.8 \%$ (7/59) of the patients, with 3 in-hospital deaths, and long-term mortality was observed in $18.64 \%(11 / 59)$.

The results regarding the association between the cardiac biomarkers and the short-term adverse events are provided in Figure 1, which illustrates the logarithmic distribution of NT-proBNP and GDF15 as well as the distribution of H-FABP and tenascin-C values in the patients with versus those without a short-term adverse outcome. Moreover, Table 2 presents the association between these biomarkers and the short-term adverse events.

\section{Value of NT-proBNP compared with other cardiac markers for the short-term outcome after acute pulmonary embolism}

As the $\operatorname{logNT}$-proBNP provided the best fit, this variable was used for analysis. The mean value of $\operatorname{logNT}-$ proBNP was $6.40( \pm 1.66) \mathrm{pg} / \mathrm{ml}$ ranging from 3.64 to $9.46 \mathrm{pg} / \mathrm{ml}$. In the patients with short-term adverse outcomes, the levels of logNT-proBNP were significantly higher than those of the patients without short-term adverse events $(7.88 \pm 0.67 \mathrm{pg} / \mathrm{ml}$ vs $6.22 \pm 1.66 \mathrm{pg} / \mathrm{ml} ; \mathrm{p}=0.041)$ (Figure 1). After adjustment for cigarette smoking, $\mathrm{O}_{2}$ saturation lower than $90 \%$, RV dysfunction, and incomplete right bundle branch block - a threefold increase in the risk of short-term adverse events was identified (OR, 3.239 ; $95 \% \mathrm{CI}, 0.877$ to $11.967 ; \mathrm{p}=0.078$ ).
The logarithm of GDF-15 was used for analysis. The mean value of $\operatorname{logGDF}-15$ was $8.17( \pm 0.57) \mathrm{pg} / \mathrm{ml}$ (range from 7.10 to $9.59 \mathrm{pg} / \mathrm{ml})$. The baseline concentration of H-FABP ranged from 1.25 to $33.30 \mathrm{ng} / \mathrm{ml}$, with a median value of $3.10 \mathrm{ng} / \mathrm{ml}$ ( $25^{\text {th }}$ to $75^{\text {th }}$ percentiles: 1.25 to 4.30 ). The median value of tenascin-C was 10.56 (4.98 to 24.52$) \mathrm{ng} / \mathrm{ml}$, ranging from 0.24 to $32.19 \mathrm{ng} / \mathrm{ml}$. The median of D-dimer was $4.00 \mathrm{mg} / \mathrm{l}$ ( $25^{\text {th }}$ to $75^{\text {th }}$ percentile: 3.600 to 4.500 ) in the patients with a complicated shortterm outcome and $5.400 \mathrm{mg} / \mathrm{l}$ ( $25^{\text {th }}$ to $75^{\text {th }}$ percentile: 1.990 to 8.200$)$ in the uncomplicated group.

The levels of GDF-15 did not influence the short-term adverse outcome, before and after adjustment for the confounders (Table 2). According to the H-FABP and Tenascin-C levels no significant differences between the two PE subgroups with and without a short-term adverse outcome revealed (Table 2). Moreover, no difference in the short-term adverse outcome was observed between the two mentioned groups according to the D-dimer levels (Table 2).

\section{Value of the markers for long-term mortality}

Long-term follow-up data were available for all the study participants. There were 11 (18.64\%) deaths in total over a follow-up period of 17 months. The unadjusted hazard ratio for logNT-proBNP was 1.578 (95\% CI, 1.017 to 2.448; $\mathrm{p}=0.042$ ). After adjustment for age, systolic blood pressure, hypertension, and immobility - the hazard ratio was 2.163 (95\% CI, 0.910 to 5.142; $\mathrm{p}=0.081)$. In this study, adjustment revealed evidence regarding an association between increased logNTproBNP levels and long-term mortality (Table 3 ). 
Table 2. Association of biomarkers with short-term adverse events in acute normotensive pulmonary embolism.

\begin{tabular}{|c|c|c|c|c|c|c|}
\hline \multirow[b]{2}{*}{ Biomarkers } & \multicolumn{3}{|c|}{ Unadjusted } & \multicolumn{3}{|c|}{ Adjusted } \\
\hline & OR & 95\% CI & p-value & OR & 95\% CI & p-value \\
\hline Log NT-proBNP & 2.359 & $1.037-5.367$ & 0.041 & 3.239 & $0.877-11.967$ & 0.078 \\
\hline Log GDF-15 & 1.255 & $0.315-4.994$ & 0.748 & 0.891 & $0.156-5.080$ & 0.897 \\
\hline H-FABP & 1.051 & $0.734-1.506$ & 0.785 & 0.925 & $0.582-1.472$ & 0.743 \\
\hline Tenascin-C & 1.013 & $0.941-1.091$ & 0.724 & 0.949 & $0.853-1.056$ & 0.338 \\
\hline D-dimer & 0.895 & $0.695-1.152$ & 0.388 & 0.880 & $0.645-1.200$ & 0.418 \\
\hline
\end{tabular}

NT-pro BNP, N-terminal pro-brain natriuretic peptide; H-FABP, heart type fatty acid-binding protein; GDF-15, growth differentiation factor-15. The odds ratios adjusted for cigarette smoking, 02 saturation $<90 \%$, right ventricular dysfunction, incomplete right bundle branch block.
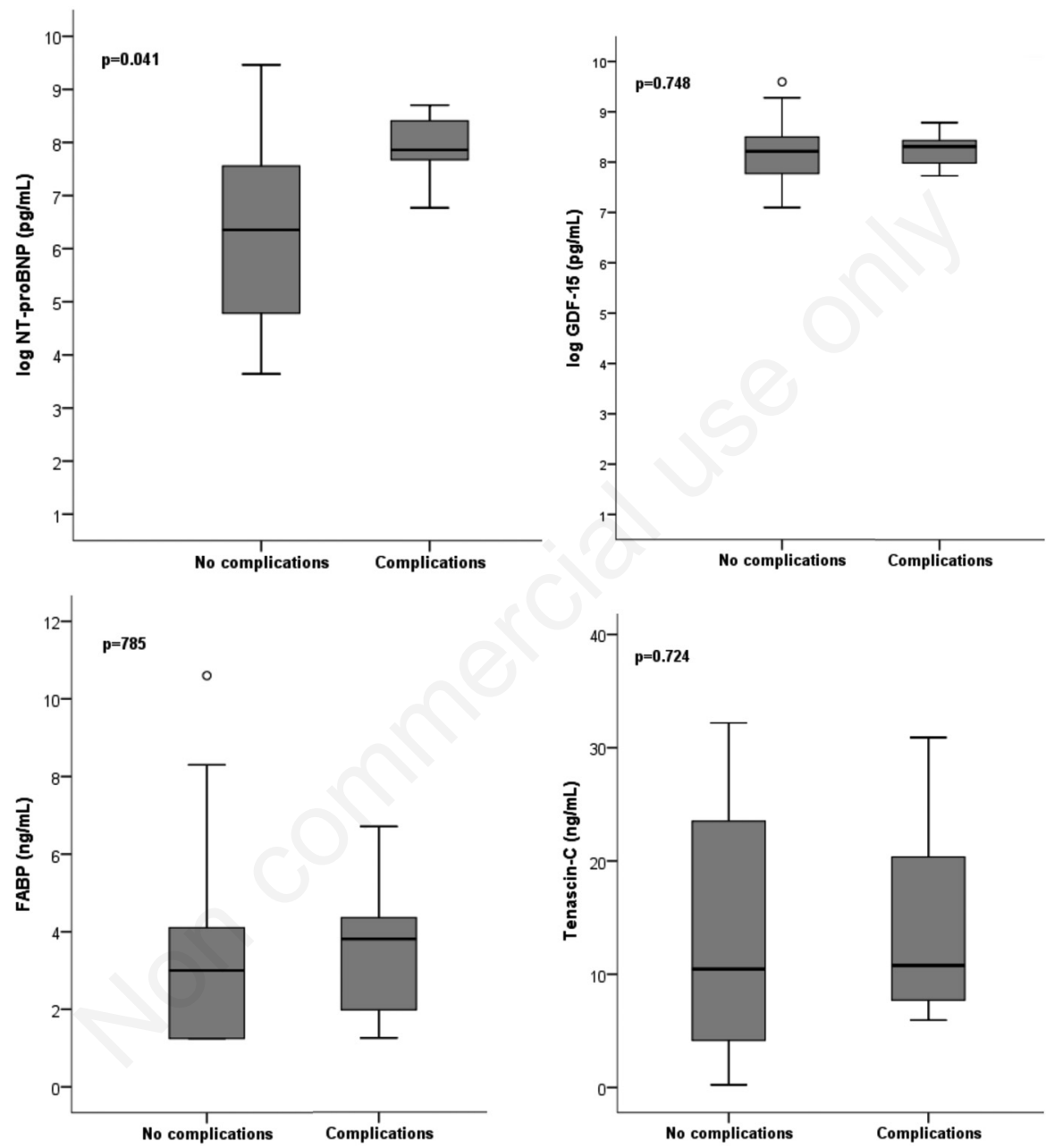

Figure 1. Logarithmic distribution of NT-proBNP, GDF-15, H-FABP and tenascin-C values in the patients with versus those without a short-term adverse outcome.

Table 3. Association of biomarkers with long-term outcome in acute normotensive pulmonary embolism.

\begin{tabular}{|c|c|c|c|c|c|c|}
\hline & & Unadjusted & & Adjuster & & \\
\hline Biomarkers & HR & 95\% CI & p-value & HR & $95 \%$ CI & p-value \\
\hline Log NT-proBNP & 1.578 & $1.017-2.448$ & 0.042 & 2.163 & $0.910-5.142$ & 0.081 \\
\hline Log GDF-15 & 2.775 & $1.036-7.435$ & 0.042 & 16.242 & $0.277-951.003$ & 0.179 \\
\hline H-FABP & 1.226 & $0.970-1.550$ & 0.089 & 0.665 & $0.350-1.265$ & 0.213 \\
\hline Tenascin-C & 1.075 & $1.015-1.137$ & 0.013 & 1.002 & $0.899-1.116$ & 0.975 \\
\hline D-dimer & 1.028 & $0.931-1.136$ & 0.586 & 0.970 & $0.802-1.174$ & 0.757 \\
\hline
\end{tabular}

The hazard ratios adjusted for age, systolic blood pressure, hypertension and immobility. 


\section{Value of the markers for RV dysfunction}

Levels of $\operatorname{logNT}$-proBNP and H-FABP were significantly higher in the patients with $\mathrm{RV}$ dysfunction $(\mathrm{P}<0.05)$. This association was not identified for tenascin-C and $\log$ GDF-15 (Table 4 ).

\section{Discussion}

The present study was conducted to identify the most important biomarkers for the prediction of short-term adverse events and/or long-term mortality in patients with normotensive acute PE. The included biomarkers of interest were NT-proBNP, GDF-15, H-FABP, tenascin-C and Ddimer. In our study patients among the examined biomarkers, only increased levels of NT-proBNP revealed evidence on association with both short-term adverse outcomes and long-term all-cause mortality.

Natriuretic peptides are stretch-related markers released from the myocytes of the ventricles upon stretch. In acute PE elevation of BNP is thought to be caused by an increase in RV strain as a consequence of the sudden occlusion of the pulmonary artery and pulmonary hypertension [1,8-10]. Our results demonstrated evidence on association between elevated levels of NT-proBNP and poor short-term outcome, longterm all-cause mortality and RV dysfunction. A similar association has been reported by previous studies $[1,8,11]$, suggesting the plasma levels of BNP at admission as a marker of RV dysfunction [12], shortterm prognosis [13], and long-term respiratory prognosis in acute PE [11]. Although echocardiography is the choice for the diagnosis of RV dysfunction, NT-proBNP can be suggested as a useful and surrogate marker for the loss of RV function especially for rapid risk stratification in the emergency department. This assumption is further supported by the results of a study which reported that only natriuretic peptides had a relationship with RV dysfunction on chest CT [14].

The cardiac expression of GDF-15 has been identified after pressure overload or myocardial ischemia. In the present study, there was a trend toward higher long-term all-cause mortality and development of RV dysfunction rather than the short-term adverse outcome in the patients with increased GDF-15. Lankeit et al. [15] assessed the prognostic value of GDF-15 in acute PE and concluded that the levels of GDF-15 on admission could help to identify patients at risk of death or serious complications and also those with a poor long-term prognosis. Our different results on adverse outcomes for elevated GDF-15 may be explained by the notion that the in acute PE elevated levels of GDF-15 may be related not only to an acute RV overload but also to underlying comorbidities such as history of underlying heart failure, renal insufficiency, or malignancy which were excluded from our study. This can mean elevated levels of GDF-15 in acute PE, cannot be interpreted as exclusively due to RV overload [16].

H-FABP is postulated to leak from damaged myocytes as a result of increased cell membrane permeability overload [16]. Our study, in consistent with the findings of a study by Puls et al. [17] who suggested H-FABP as an early marker of RV dysfunction in acute PE, re- vealed an association between higher levels of H-FABP and development of RV dysfunction. A study by Vuilleumier et al. [14] suggested NT-proBNP as the strongest predictor of death and hospitalization in comparison with damage-related markers including H-FABP. Similarly, in our study patients and in the presence of NT-proBNP association was found neither with the short-term adverse events nor long-term mortality in terms of H-FABP. However, previous studies on H-FABP have suggested this marker as a promising biomarker for risk stratification in acute $\mathrm{PE}[6,15,16,18]$. These conflicting findings may be to some extent due to the relatively low number of events in our study population or the different inclusion criteria for our study.

Tenascin-C, an extracellular matrix protein, has been linked to cardiovascular diseases. To our knowledge however there is lack of information on the value of tenascin- $C$ in patients with acute PE. One of the first studies on this subject was conducted by Celik et al. (19) reported a significant increase in tenascin-C levels in thirty-four PE patients who developed pulmonary hypertension. The findings of the current study did not confirm the reports by Celik et al. We found no association between increased levels of tenascin- $\mathrm{C}$ whether in short-term adverse events or in long-term mortality. This disagreement in the results may have stemmed from Celik and colleagues' different study population which studied patients with massive and submassive PE as well as patients with malignancy.

Finally, similar to the Tanabe et al. study [10], in the present study the status of D-dimer was not a good indicator for the prognosis of PE. This can be explained by the fact that D-dimer also indicates a systemic reaction to dissolving thrombus, deep vein thrombosis, and inflammatory diseases. There are only a few investigations suggesting D-dimer as a good indicator for the prognosis of PE [20-22].

Moreover, the most accurate biomarker as predictor of RV dysfunction has not been yet determined. There are evidences on different cardiac markers to have association with RV dysfunction to some extent. However, further validation is needed and at present biomarkers should be considered together with clinical risk scores for risk stratification of PE patients [14,23,24].

\section{Limitations}

There were some potential limitations to the present study. As a single-center study, the sample size could not be as large as desired. However, our adjustment analysis revealed a trend toward short-term adverse events and long-term mortality for increased NT-proBNP. The borderline $\mathrm{P}$ values were also partly a result of the relatively low number of adverse events in our study. Accordingly, our results should be considered preliminary and further trials on large populations are required to assess the prognostic value of such biomarkers in practice.

The salient points of the current study are the evaluation of different novel cardiac biomarkers, long-term follow-up of the patients, and exclusion of patients with comorbidities to prevent their effect on the interpretation of the results of the markers.

Table 4. Association of biomarkers with right ventricular dysfunction in acute pulmonary embolism.

$\begin{array}{lccc}\text { Biomarkers } & \text { With RVD } & \text { Without RVD } & \text { p-value } \\ & (\mathrm{n}=31) & 5.22( \pm 1.29) & <0.001 \\ \text { Log NT-proBNP } & 7.46( \pm 1.18) & 8.07( \pm 0.61) & 0.177 \\ \text { Log GDF-15 } & 8.27( \pm 0.54) & 2.800(1.250-3.800) & 0.043 \\ \text { H-FABP } & 3.600(1.250-5.750) & 10.095(2.867-21.877) & 0.316 \\ \text { Tenascin-C } & 10.950(5.83-26.46) & 4.330(1.420-8.732) & 0.379 \\ \text { D-dimer } & 5.450(3.900-6.960) & \end{array}$

Data are presented as median, $25^{\text {th }}-75^{\text {th }}$ percentile or mean \pm standard deviation. 


\section{Conclusions}

Among studied biomarkers, our observational study could find evidences on association between increased level of NT-proBNP and higher risk of either short-term adverse events or long-term mortality and RV dysfunction in acute PE. Nonetheless, whether the elevation of GDF-15, H-FABP and tenascin-C, compared with NT-proBNP can be considered a predictor of the outcome in patients with acute PE has yet to be determined by further large sample studies.

\section{References}

1. Verschuren F, Bonnet M, Benoit M0, et al. The prognostic value of pro-B-Type natriuretic peptide in acute pulmonary embolism. Thromb Res 2013;131:e235-e239.

2. Kearon C, Kahn SR, Agnelli G, et al. Antithrombotic therapy for venous thromboembolic disease: American College of Chest Physicians evidence-based clinical practice guidelines. Chest 2008;133:S454S-545.

3. Jimenez D, Yusen RD. Prognostic models for selecting patients with acute pulmonary embolism for initial outpatient therapy. Curr Opin Pulm Med2008;14:414-21.

4. Konstantinides SV, Torbicki A, Agnelli G, et al. 2014 ESC Guidelines on the diagnosis and management of acute pulmonary embolism. Eur Heart J 2014;35:3033-69.

5. Klok FA, Mos IC, Huisman MV. Brain-type natriuretic peptide levels in the prediction of adverse outcome in patients with pulmonary embolism: a systematic review and meta-analysis. Am J Respir Crit Care Med 2008;178:425-30.

6. Kaczynska A, Pelsers MM, Bochowicz A, et al. Plasma heart-type fatty acid binding protein is superior to troponin and myoglobin for rapid risk stratification in acute pulmonary embolism. Clin Chim Acta 2006;371:117-23.

7. Tapson VF. Acute pulmonary embolism. N Engl J Med 2008;358: 1037-52.

8. Krüger S, Graf J, Merx MW, et al. Brain natriuretic peptide predicts right heart failure in patients with acute pulmonary embolism. Am Heart J 2004;147:60-5.

9. Sagcan G, Arseven 0, Okumus G, et al. Importance of cardiac biomarkers in the evaluation of acute pulmonary thromboembolism severity, mortality and complicated clinical course. Eur Respir J 2011;38:p3610.

10. Tanabe Y, Obayashi T, Yamamoto T, et al. Predictive value of biomarkers for the prognosis of acute pulmonary embolism in Japanese patients: Results of the Tokyo CCU Network registry. J Cardiol 2015;66:460-5.

11. Ohigashi H, Haraguchi G, Yoshikawa S, et al. Comparison of biomarkers for predicting disease severity and long-term respiratory prognosis in patients with acute pulmonary embolism. Int Heart $\mathrm{J}$ 2010;51:416-20.

12. Kostrubiec M, Pruszczyk P, Kaczynska A, Kucher N. Persistent NTproBNP elevation in acute pulmonary embolism predicts early death. Clin Chim Acta 2007;382:124-8.

13. Pruszczyk P, Kostrubiec M, Bochowicz A, et al. N-terminal pro-brain natriuretic peptide in patients with acute pulmonary embolism. Eur Respir J 2003;22:649-53.

14. Vuilleumier N, Le Gal G, Verschuren F, et al. Cardiac biomarkers for risk stratification in non-massive pulmonary embolism: a multicenter prospective study. J Thromb Haemost 2009;7:391-8.

15. Lankeit M, Kempf T, Dellas C, et al. Growth differentiation factor15 for prognostic assessment of patients with acute pulmonary embolism. Am J Respir Crit Care Med 2008;177:1018-25.

16. Lauque D, Maupas-Schwalm F, Bounes V, et al. Predictive value of the heart-type fatty acid-binding protein and the pulmonary embolism severity index in patients with acute pulmonary embolism in the emergency department. Acad Emerg Med 2014;21:1143-50.

17. Puls M, Dellas C, Lankeit M, et al. Heart-type fatty acid-binding protein permits early risk stratification of pulmonary embolism. Eur Heart J 2007;28:224-9.

18. Boscheri A, Wunderlich C, Langer M, et al. Correlation of heart-type fatty acid-binding protein with mortality and echocardiographic data in patients with pulmonary embolism at intermediate risk. Am Heart J 2010;160:294-300.

19. Celik A, Kocyigit I, Calapkorur B, et al. Tenascin-C may be a predictor of acute pulmonary thromboembolism. J Atheroscler Thromb 2011;18:487-93.

20. Becattini C, Lignani A, Masotti L, et al. D-dimer for risk stratification in patients with acute pulmonary embolism. J Thromb Thrombolysis 2012;33:48-57.

21. Lobo J, Zorrilla V, Aizpuru F, et al. D-dimer levels and 15-day outcome in acute pulmonary embolism. Findings from the RIETE Registry. J Thromb Haemost 2009;7:1795-801.

22. Aujesky D, Roy P, Guy M, et al. Prognostic value of D-dimer in patients with pulmonary embolism. Thromb Haemost 2006;96:478-82.

23. Kucher N, Goldhaber SZ. Cardiac biomarkers for risk stratification of patients with acute pulmonary embolism. Circulation 2003;108: 2191-4.

24. Giannitsis E, Katus HA. Biomarkers for clinical decision-making in the management of pulmonary embolism. Clin Chem 2016; 63:91-100. 